\title{
Modélisation des ressources manganésifères de la zone B-Centre (gisement de manganèse de Lauzoua, Côte d'Ivoire)
}

\author{
Niangoran Kouadio Charles, \\ Bie Goha René, \\ Gbangbot Jean-Michel Kouadio, \\ Université Jean Lorougnon Guédé, Laboratoire de Science et Technologie de \\ l'Environnement Daloa, Côte d'Ivoire
}

\section{Résumé}

La construction de modèles 3D et leur utilisation demeurent répandues dans le domaine de la géologie. Cela peut être expliqué, entre autres, par la diversité des données à disposition et les nombreuses étapes à réaliser afin de construire un modèle $3 \mathrm{D}$ valide et cohérent par rapport à son utilisation. Afin de pallier ce déficit au sein de la CML, ce projet d'étude a été mené dans un contexte particulier. En effet, la minéralisation manganésifère de la mine de Lauzoua est de très faible épaisseur ( 8 à $13 \mathrm{~m}$ ) contrairement aux autres mines dans le monde. De ce fait la mine de Lauzoua est exploitée sous forme de carrière. Les projets avancés s'arrêtent donc à l'étape des puits et tranchées. Les tables de données pour la modélisation sont donc des données issues des puits de prospection et non de sondages. Les données sont entre autres l'identifiant du puits, les coordonnées (X, Y, Z), les épaisseurs échantillonnées et les résultats d'analyse géochimiques (teneurs en $\mathrm{Mn}$ ). Les tables ont été utilisées pour créer un modèle solide puis validé. Les ressources ont été estimées par trois méthodes d'interpolation à savoir le krigeage, la méthode $\mathrm{du}$ plus proche voisin et la pondération inverse de la distance. Les tests statistiques ont permis de retenir l'estimation faite par le krigeage ordinaire, soit $559411 \mathrm{~T}$ de minerai d'une teneur moyenne de $29,44 \%$. La modélisation permet de réduire les coûts d'exploration et d'exploitation des gisements. Ensuite, elle contribue à réduire les risques financier et matériel. Enfin, elle permet une meilleure planification des opérations d'extraction. C'est un outil d'aide à la prise de décision.

Mots-clés : Modélisation, ressources, gisement, manganèse, CML 


\title{
Modeling of Manganiferous Resources in the B-Centre zone (Lauzoua Manganese Deposit Côte d'Ivoire)
}

\author{
Niangoran Kouadio Charles, \\ Bie Goha René, \\ Gbangbot Jean-Michel Kouadio,
} Université Jean Lorougnon Guédé, Laboratoire de Science et Technologie de l'Environnement Daloa, Côte d'Ivoire

\begin{abstract}
The construction of 3D models and their use remain uncommon in the field of geology. This can be explained, among other things, by the diversity of the data available and the many steps to be taken in order to build a valid $3 \mathrm{D}$ model that is consistent with its use. In order to overcome this deficit within CML, this study project was carried out in a particular context. Indeed, the manganesiferous mineralization of the Lauzoua mine is very thin ( 8 to 13 $\mathrm{m})$ compared to mines in the world, and as a result is exploited in the form of a quarry, so advanced projects stop at the shaft and trench stage. The data tables for modelling are therefore data from prospecting wells and not from boreholes. The data include well ID, coordinates (X, Y, Z), sampled thicknesses, geochemical analysis results (Mn values), etc. The tables were used to create a solid model and then validated. Resources were estimated by three interpolation methods. Statistical tests made it possible to retain the estimate made by ordinary kriging, i.e. 559411T of ore with an average grade of $29.44 \%$. Modelling reduces the costs of exploration and exploitation of deposits and reduces financial and material risks. It allows a better planning of extraction operations, it is a decision-making tool.
\end{abstract}

Keywords: Modeling, resources, deposit, manganese, CML

\section{Introduction}

Le métal manganèse $(\mathrm{Mn})$ est relativement abondant dans la croûte terrestre où il occupe la $12^{\text {ième }}$ place avec un Clarke de $0.1 \%$ (BRGM, 1984). Le manganèse est l'élément essentiel à la production de l'acier (90\%) grâce à sa capacité à fixer le soufre, sa propriété anti-oxydante et son aptitude à la formation d'alliages. 
Avec l'appétit insatiable de la Chine, même si elle occupe le pôle des pays producteur, et l'émergence des pays en voie de développement (Brésil, Japon, Inde, Afrique du Sud), la demande de manganèse ne fait que crôitre. C'est pourquoi en Côte d'Ivoire, en 1994, la SODEMI a repris les études de l'ancienne mine de Mokta et a évalué les ressources à 1631.086 tonnes à 41,97 $\% \mathrm{Mn}$. La production ivoirienne est exclusivement destinée à l'exportation. Jusque-là, le processus d'estimation des ressources adopté par la Compagnie Minière du Littoral (CML) est basé sur des modèles 2D. La carte 2D est un modèle bidimensionnel de la réalité, car, elle est une représentation de l'environnement géologique naturellement tridimensionnel sur un média ayant deux dimensions. Les inconvénients à l'utilisation des cartes 2D sont tels que, d'une part comme la minéralisation n'est représentée que sur un plan, tout le reste du sous-sol peut être interprété de diverses façons par différents spécialistes. D'autres parts, les cartes 2D sont des simplifications de la réalité qui ne représentent qu'une interprétation d'un spécialiste et elles ne représentent pas toutes les données d'origine. Enfin, il peut être difficile d'interpréter correctement la géologie lorsque l'environnement géologique est très complexe et déformé. Il faut donc s'assurer que chaque géologue qui utilise ces modèles 2D, créés par un autre géologue, interprète correctement en trois dimensions ce qu'il voit sur ces modèles 2D (Brodaric et al., 2004). La modélisation des environnements géologiques en trois dimensions est donc certainement un objectif à atteindre pour les géologues. On peut ainsi représenter de manière plus exhaustive des objets qui sont en $3 \mathrm{D}$ et obtenir un modèle plus près de la réalité. C'est dans ce contexte qu'a été initiée la présente étude de modélisation des ressources manganésifères de la zone BCentre (gisement de manganèse de Lauzoua). L'objectif général de cette étude est de construire le modèle géologique 3D du gisement de la zone B-Centre et d'évaluer ces ressources manganésifères.

\section{Localisation géographique du gisement de manganèse de Lauzoua}

Le gisement de manganèse de Lauzoua se situe au Sud de la Côte d'Ivoire précisément à la lisière du bassin sédimentaire, dans le département de Guitry et dans la sous-préfecture de Lauzoua. Il est situé à environ $53 \mathrm{~km}$ au nord-ouest de Grand-Lahou dont $7 \mathrm{~km}$ de piste entre la route « côtière » et l'ancienne mine de Mokta. Il est couvert par le permis d'exploitation PE 36 d'une superficie de $100 \mathrm{~km}^{2}$ (Figure 1) et le nouveau permis de recherche PR 248 d'une superficie de 88,6 km² (Dago, 2014). 


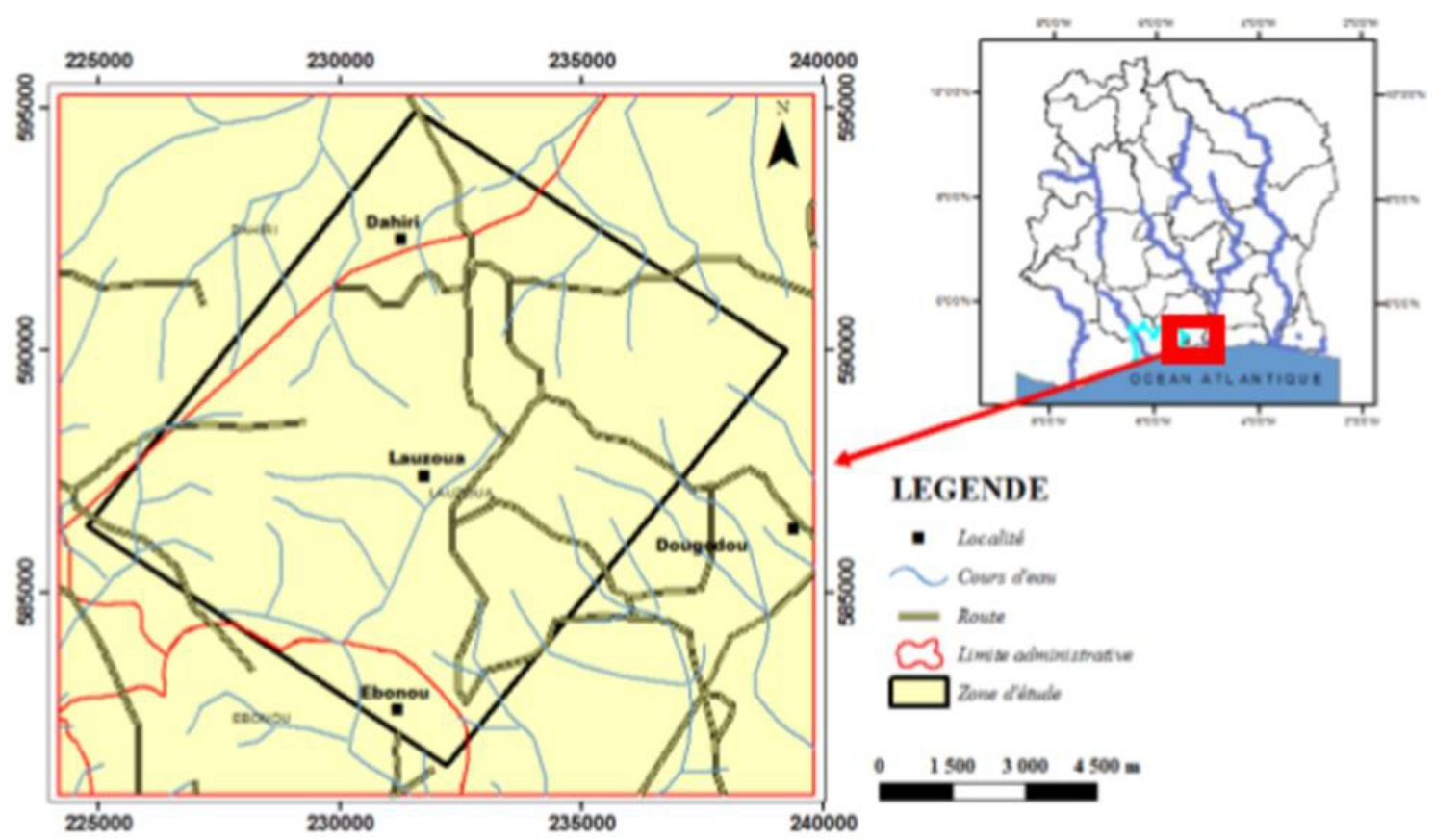

Figure 1: Carte de localisation du permis d'exploitation de Lauzoua

\section{Contexte géologique du Gisement de Lauzoua}

On peut distinguer dans la région de Lauzoua, des formations métamorphiques d'origine sédimentaire, les formations métamorphiques d'origine volcanique et les formations volcaniques (Papon, 1962). Le manganèse se situe dans une bande étroite limitée à l'Ouest par les granites syntectoniques, à l'est par le domaine schisteux de la basse Comoé et au Sud par le bassin sédimentaire. Cette bande disparaît vers le nord, à la hauteur de Tiassalé contre des granites syntectoniques. L'horizon manganésifère est interstratifié dans les schistes en une série de lentilles discontinues les unes à la suite des autres selon la direction birimienne (NE-SW). (Figure 2). 


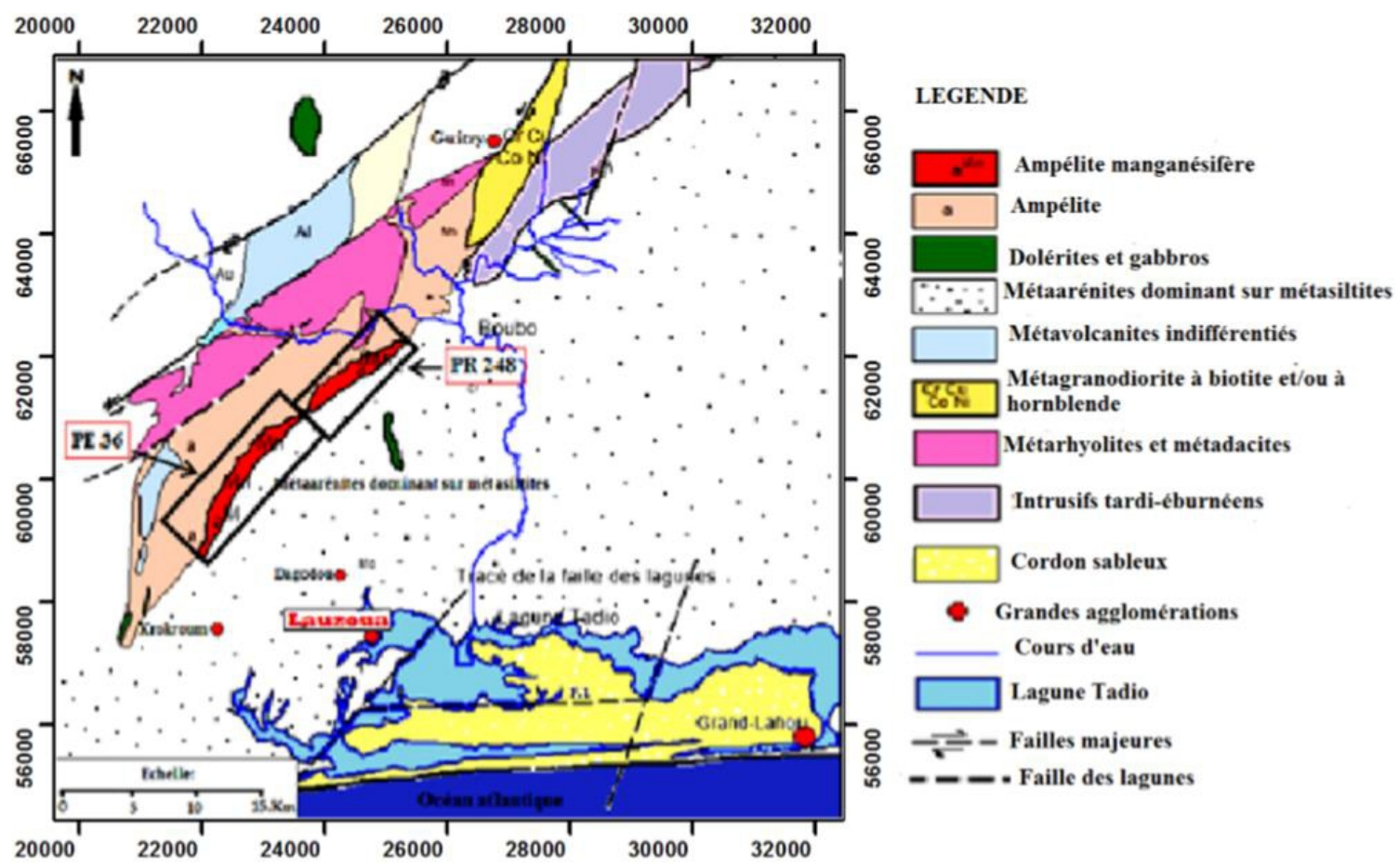

Figure 2 : Carte géologique de la région Lauzoua (Delor et al., 1995)

\section{Matériel et méthodes}

\subsection{Données de l'étude}

Comprendre la distribution géochimique du gisement implique la connaissance du profil des puits. Dès lors, les données utilisées à cet effet sont:

- les données d'analyses géochimiques des épaisseurs minéralisées (teneur en Mn) ;

- les données d'identité des puits de prospection (X, Y, Z, pendage et azimut) ;

- les données de densité des épaisseurs minéralisées ;

- les données du suivi des directions et des pendages des puits de prospection.

\subsection{Méthodes}

\subsubsection{Processus de création, standardisation et de correction de la base} de données

Elle constitue l'étape préliminaire avant la modélisation proprement dite. Les données de base ont été stockées dans quatre tables Excel (xls) enregistrées sous format Excel (CSV). Les quatre tables de données de base sont liées entre elles par l'identifiant des puits. La base de données ainsi obtenue a été corrigée afin d'éliminer les enregistrements redondants et 
erronés pour une utilisation efficace lors de l'élaboration du modèle géologique.

\subsubsection{Elaboration de modèle géologique}

Sur la base des données enregistrées dans la table Collar, les 374 puits sont positionnés dans le graphique 3D. La table Collar correspondant au positionnement $(\mathrm{X}, \mathrm{Y}, \mathrm{Z}) \mathrm{du}$ puits, sa profondeur, son pendage et son azimut.

Les puits ont été liés en sélectionnant une section parallèle à la direction de meilleure distribution (NE-SW) de logs et ensuite digitalisés en tenant compte des paramètres d'analyses géochimiques (teneur en $\mathrm{Mn}$ ) pour former des chaines de minerai. Les sections parallèles ont été nettoyées des zones de croisement ou de chevauchement et des points dupliqués. Les chaines de minerai créées ont été triangulées en les liant deux à deux pour couvrir l'intégralité du dépôt. Cette étape consiste en la première phase du modèle solide.

La validation du corps minéralisé obtenu permet de s'assurer qu'il n'y a pas d'intersection entre les triangles et que le modèle est entièrement fiable car, cela est le préalable à toute estimation des ressources. Un rapport de validation fourni par le logiciel après la requête de validation permet la vérification de la fiabilité du modèle géologique obtenu.

\subsubsection{Estimation des ressources}

L'estimation des ressources a été effectuée par trois méthodes d'interpolation à savoir le krigeage, la méthode du plus proche voisin et la pondération inverse de la distance . Toutefois, sa mise en œuvre a recouru à des étapes intermédiaires qui sont : création des composites de densité et de teneur, modélisation de bloc 3D des teneurs, attributs et contraintes.

Le composite des teneurs et celui des densités sont créés par extraction des données incluses dans l'intersection du modèle solide et des épaisseurs de puits minéralisés d'intérêt. La longueur des composite dans cette présente étude est de $1 \mathrm{~m}$. Les composites sont incontournables, car ils permettent l'uniformisation des longueurs des échantillons et l'estimation par remplissage $\mathrm{du}$ bloc modèle. Les coordonnées 3D du bloc modèle ont été définis spatialement par les étendus du modèle géologique (Figure 3).

- Maximun Northing (Y), Easting (X), et l'altitude (Z)

- Minimum Northing (Y), Easting (X) et l'altitude (Z) 


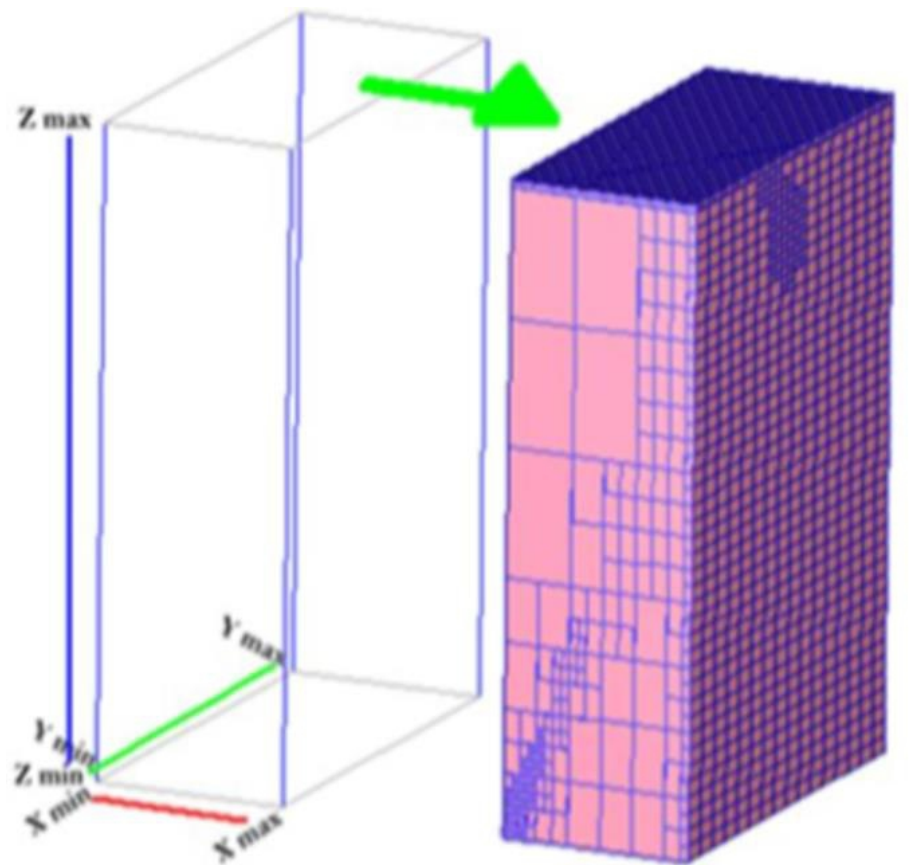

Figure 3 : Exemple de bloc modèle (Geovia Surpac)

Le bloc 3D des teneurs a fourni une méthode pour estimer le volume, le tonnage, la teneur moyenne d'un corps 3D à partir de données de puits clairsemés. Le centroîde de chaque bloc a défini ses dimensions géométriques dans chaque axe, c'est-à-dire ses coordonnées $\mathrm{Y}, \mathrm{X}$, et Z. Dans le cas spécifique de cette étude, une contrainte a été appliquée au modèle spatiale du corps minéralisé et aussi aux teneurs supérieures ou égales à $30 \%(\mathrm{Tc} \geq 30 \%)$ afin de permettre au cours de l'exploitation, une extraction sélective des zones à forte teneur.

L'estimation des ressources a été effectuée par la méthode géostatistique du krigeage ordinaire. Son utilisation a nécessitée l'étude d'un variogramme.

\subsubsection{Validation des estimations}

Il est important lors de la réalisation du modèle géologique de valider la qualité des méthodes utilisées puisqu'ils ne sont pas automatiquement représentatifs de la réalité. Afin de déterminer les méthodes d'interpolation les plus efficaces pour la représentation du gisement de la zone B-CENTRE, plusieurs indicateurs peuvent être utilisés. Ce sont la validation des points 'test' et des points 'application', l'erreur quadratique moyenne, des tests statistiques, des cartes de l'erreur,etc. Dans la présente étude, les tests statistiques ont été utilisés pour comparer les méthodes d'interpolation deux à deux. Les tests statistiques ont permis de déterminer les plus performantes. Ce 
sont celles qui présentent un coefficient de corrélation élevé et un rapport faible.

\section{Résultats et discussion}

\subsection{Résultats}

\subsubsection{Corps minéralisé triangulé}

Les chaines de minerai ont été triangulées pour former le modèle solide du dépôt de la zone B-centre puis validé en le nettoyant de toutes erreurs éventuelles. L'objet ainsi obtenu est appelé modèle solide. Il est constitué de trois lentilles indépendantes d'orientation générale NE-SW et de faible pendage (9 à $15 \%$ ) et d'une épaisseur maximale de $13 \mathrm{~m}$ (Figure 5).
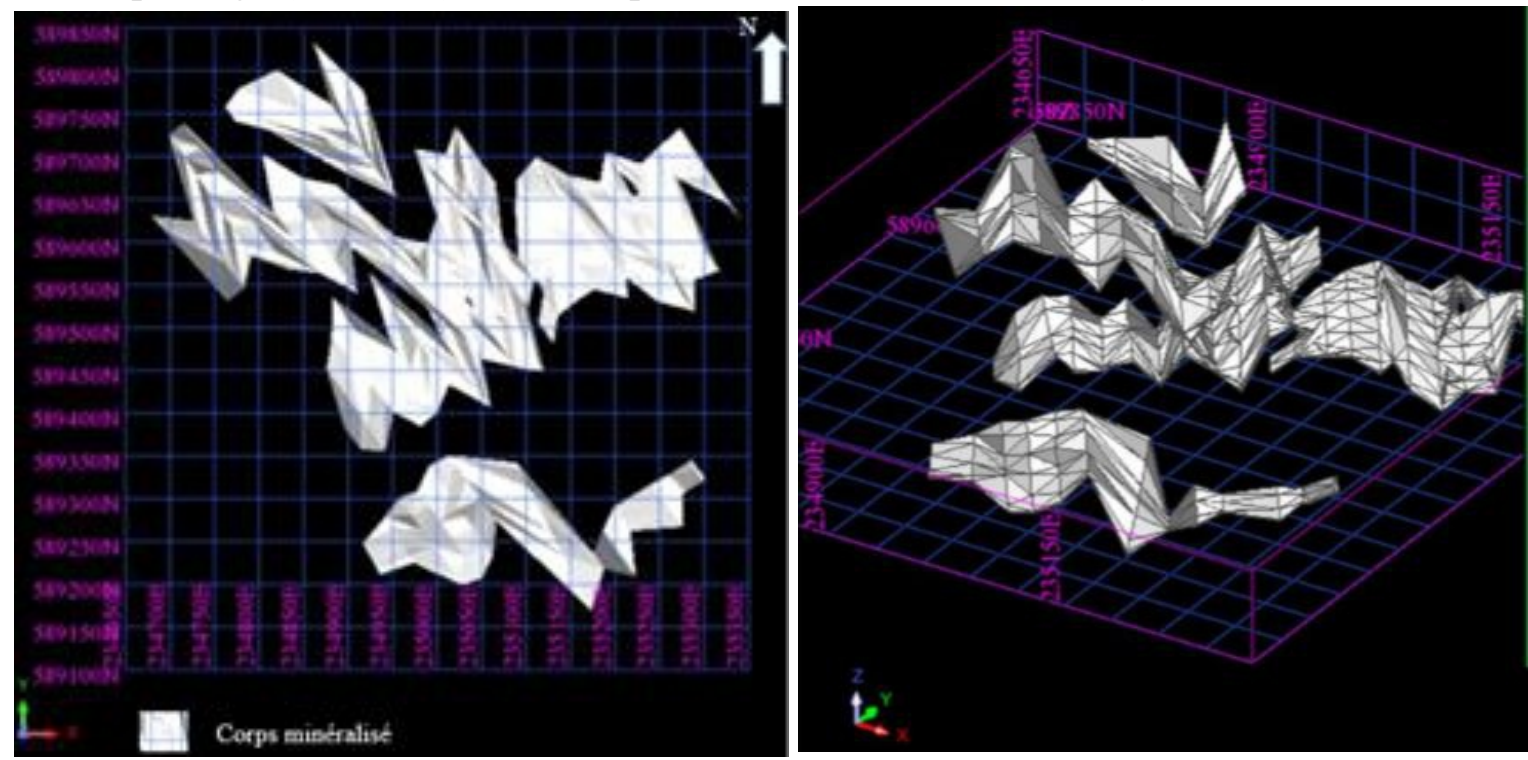

Figure 5 : Gisement modélisé de la zone B-CENTRE corps minéralisés vue du ciel (A) ; corps minéralisés vue en perspective avec triangulation visible (B).

\subsubsection{Composite des densités et des teneurs}

\subsubsection{Composite des densités}

Le composite des densités constitué à chaque mètre a donné 421 échantillons pour l'ensemble des 374 puits de prospection. L'histogramme du composite de densité est présenté à la figure 6. L'analyse statistique du composite de densité (Tableau II) a permis de connaitre la distribution statistique des densités. On remarque que la moyenne de densité des échantillons prélevés est de 1,77 et que les valeurs autour de la moyenne varient très peu car, la variance est de 0,07 . Seulement $2,3 \%$ des échantillons ont une valeur de densité inférieure ou égale à 1,4. 


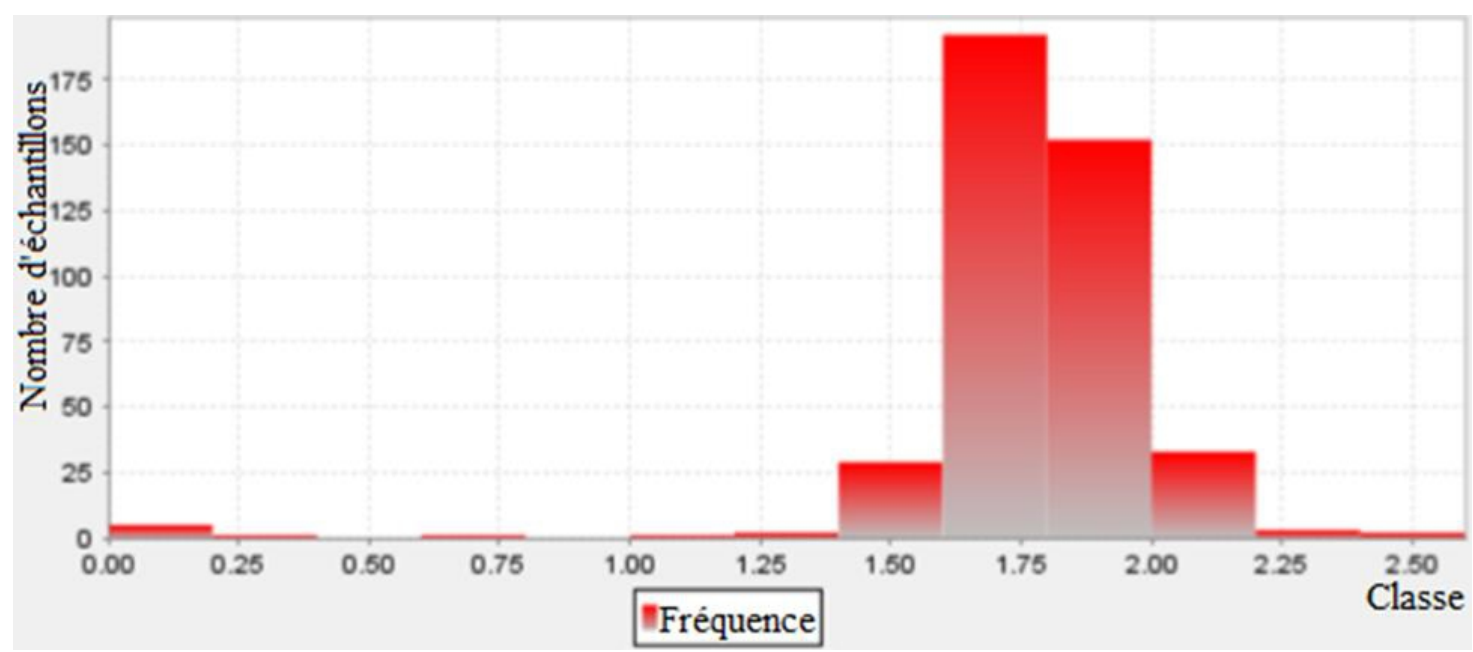

Figure 6 : Histogramme du composite des densités

Tableau III : Statistique du composite des teneurs

\begin{tabular}{|l|c|}
\hline Paramètres statistiques & \\
\hline Nombre d'échantillons du composite & 421 \\
\hline Teneur minimum () & 0 \\
\hline Teneur maximale & 2,56 \\
\hline Moyenne & 1,77 \\
\hline Médiane & 1,78 \\
\hline Variance & 0,15 \\
\hline Coefficient de variantion & 0,07 \\
\hline Ecart-type & 0,26 \\
\hline
\end{tabular}

\subsubsection{Composite des teneurs}

Le composite des teneurs constitué à chaque mètre a donné 860 échantillons pour l'ensemble des 374 puits de prospection. L'histogramme du composite des teneurs est présenté à la figure 7 . L'analyse statistique du composite des teneurs (Tableau III) a permis de connaitre la distribution statistique des teneurs. On remarque que la moyenne des teneurs des échantillons prélevés est de $28,27 \%$ et que les valeurs autour de la moyenne varient énormément car, la variance est de 138,85. Seulement 15\% (131 échantillons) des 860 échantillons ont une valeur de teneur inférieure ou égale à $14 \%$. 


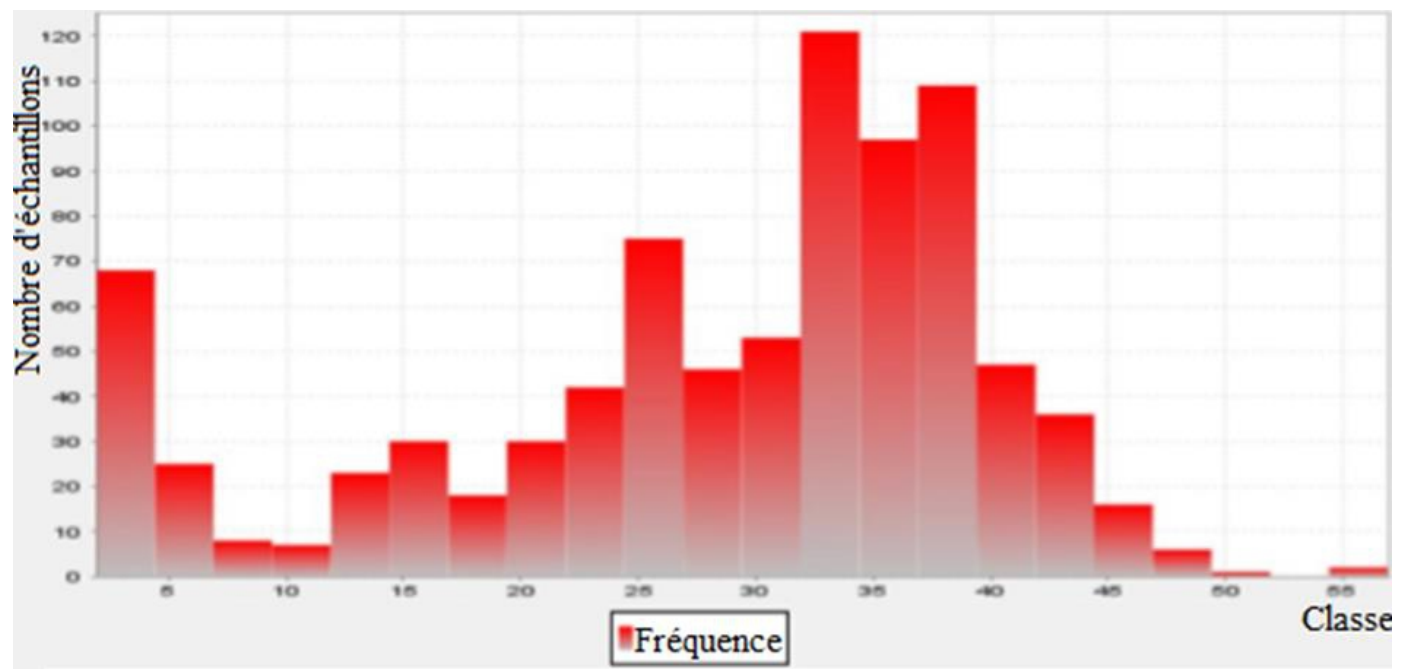

Figure 7 : Histogramme de composite des teneurs

Tableau III : Statistique du composite des teneurs

\begin{tabular}{|l|c|}
\hline Paramètres statistiques & \\
\hline Nombre d'échantillons du composite & 86 \\
\hline Teneur minimum g/t & 1,90 \\
\hline Teneur maximale g/t & 54,55 \\
\hline Moyenne & 28,27 \\
\hline Médiane & 32,08 \\
\hline Variance & 138,85 \\
\hline Coefficient de variance & 0,42 \\
\hline Ecart-type & 11,78 \\
\hline
\end{tabular}

\subsubsection{Conception de Bloc modèle des teneurs}

\subsubsection{Espace modèle}

Les coordonnées du bloc modèle ont été définies spatialement comme indiqué au tableau IV et les dimensions de chaque sous bloc adoptées sont $25 \mathrm{mx} 25 \mathrm{~m} \times 1 \mathrm{~m}$ et les sous blocs de dimensions variables sont de $12,5 \mathrm{mx} 12,5$ $\mathrm{m} \times 0,5 \mathrm{~m}$ soit au total 2380 sous blocs (Figure 8 ).

Tableau IV : Coordonnées du bloc modèle

\begin{tabular}{|c|c|c|c|}
\cline { 2 - 4 } \multicolumn{1}{c|}{} & Latitude & Longitude & Altitude \\
\hline Minimum (m) & 589152,059 & 23467,514 & 64,855 \\
\hline Maximum (m) & 589832,863 & 235341,165 & 128,659 \\
\hline
\end{tabular}




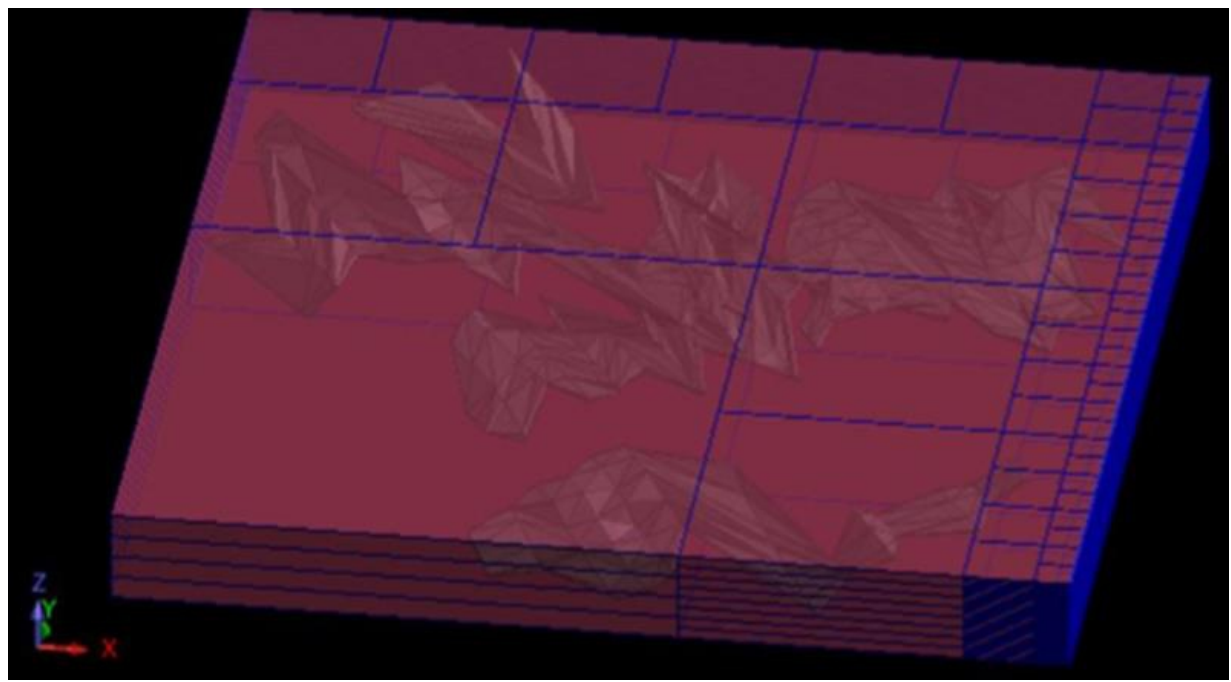

Figure 8 : Vue en perspective (azimut $352^{\circ}$, pendage $-30^{\circ}$ ) d'un bloc modèle.

\subsubsection{Contraintes}

Toutes les fonctions du bloc modèle ont été exécutées avec les paramètres spatiaux du corps minéralisé. Cela a donné un bloc modèle réduit aux coordonnées spatiales des trois lentilles (Figure 9). Les contraintes appliquées aux teneurs de coupure supérieures à $30 \%$ sont graphiquement perceptibles dans les résultats des méthodes d'interpolation.

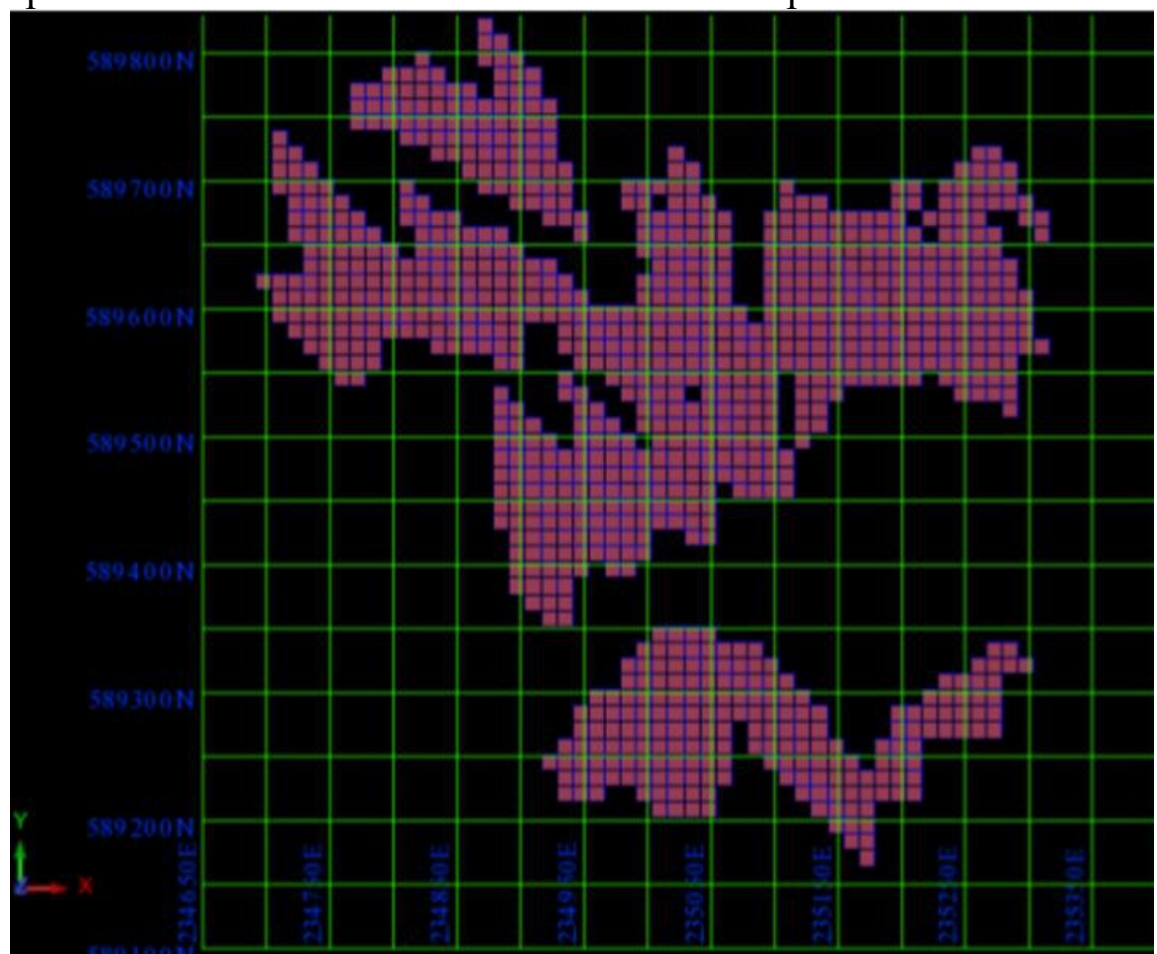

Figure 9 : Bloc modèle contraint 


\subsubsection{Analyse géostatistique et estimation des ressources.}

\subsubsection{Krigeage ordinaire.}

\subsection{Analyse du variogramme}

Le variogramme pour l'interpolation et l'estimation géostatistique du krigeage ordinaire est isotropique et de type sphérique (Figure 10). Les échantillons prélevés ne présentent quasiment pas d'effet pépite. Les paramètres variographiques obtenus sont : Effet pépite $=0,102374 ;$ Palier $=$ 0,$986403 ;$ Portée $=4,625$.

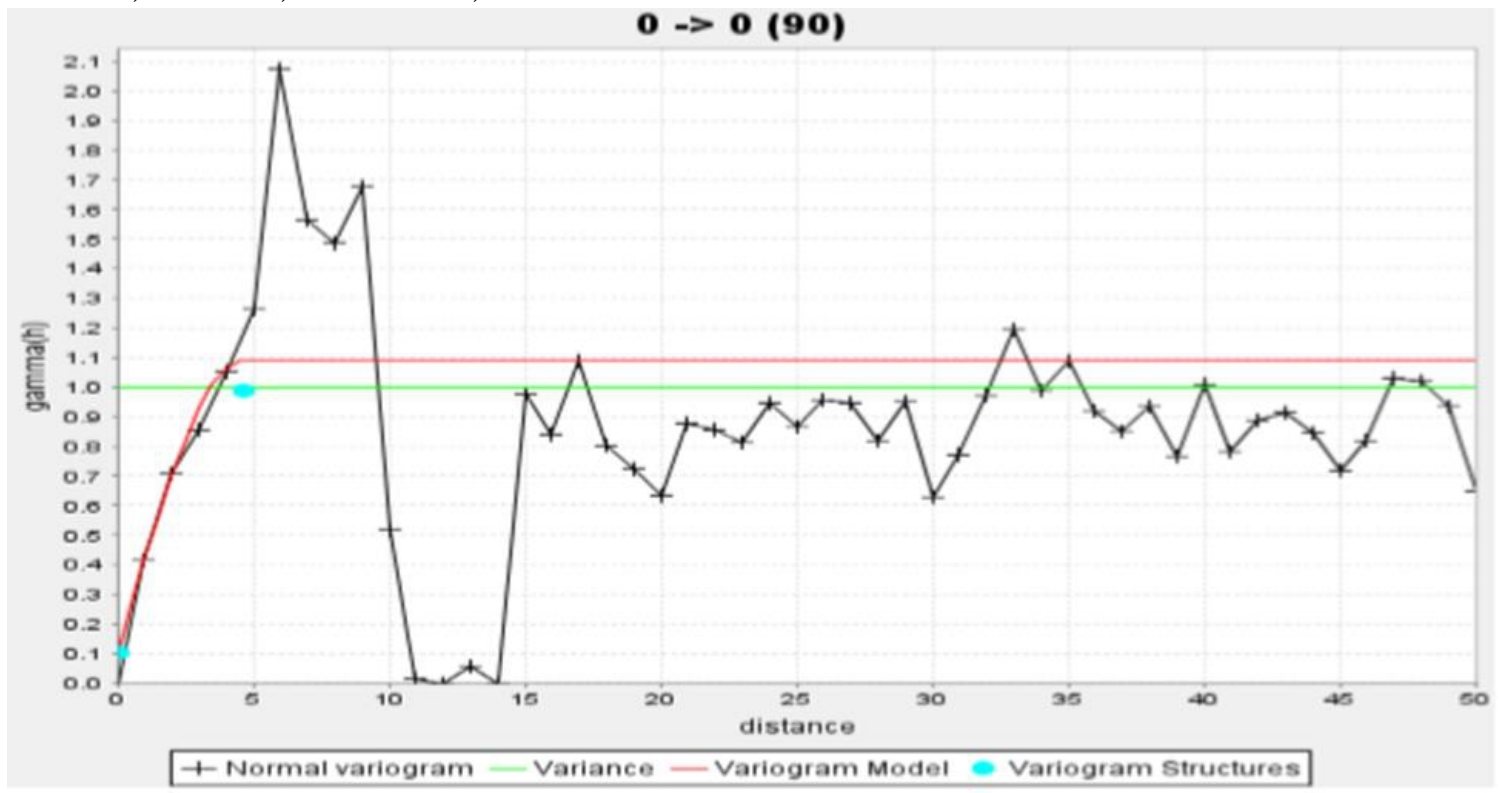

Figure 10 : Analyse variographique de la zone B-CENTRE

\subsection{Estimation des ressources par Krigeage ordinaire}

Le Krigeage ordinaire a permis d'obtenir les modèles krigés de blocs contraints des teneurs de la zone B-Centre (Figure 11). Le modèle géologique krigé présente essentiellement deux zones de teneur dont une variante du vert (19\% à 32\%) et une variante du jaune (32\% à 40\%). Les ressources ont été évaluées suivant les teneurs de coupure (Tc) comprise entre $15 \%$ et $30 \%$ pour les basses teneurs soit 291786t pour une teneur moyenne de 25,88\%. En ce qui concerne les ressources pour les hautes teneurs, seules les teneurs de coupures supérieures à $30 \%$ soit $267625 \mathrm{t}$ pour une teneur moyenne de $33,34 \%$, (Tableau V). Au total, les ressources sont estimées à 559411t pour une teneur moyenne de 29,44\%. Les modèles de blocs contraints de teneur de coupure, Tc $\geq 30 \%$ qui intéressent la CML ont été réalisés (Figure 12). 

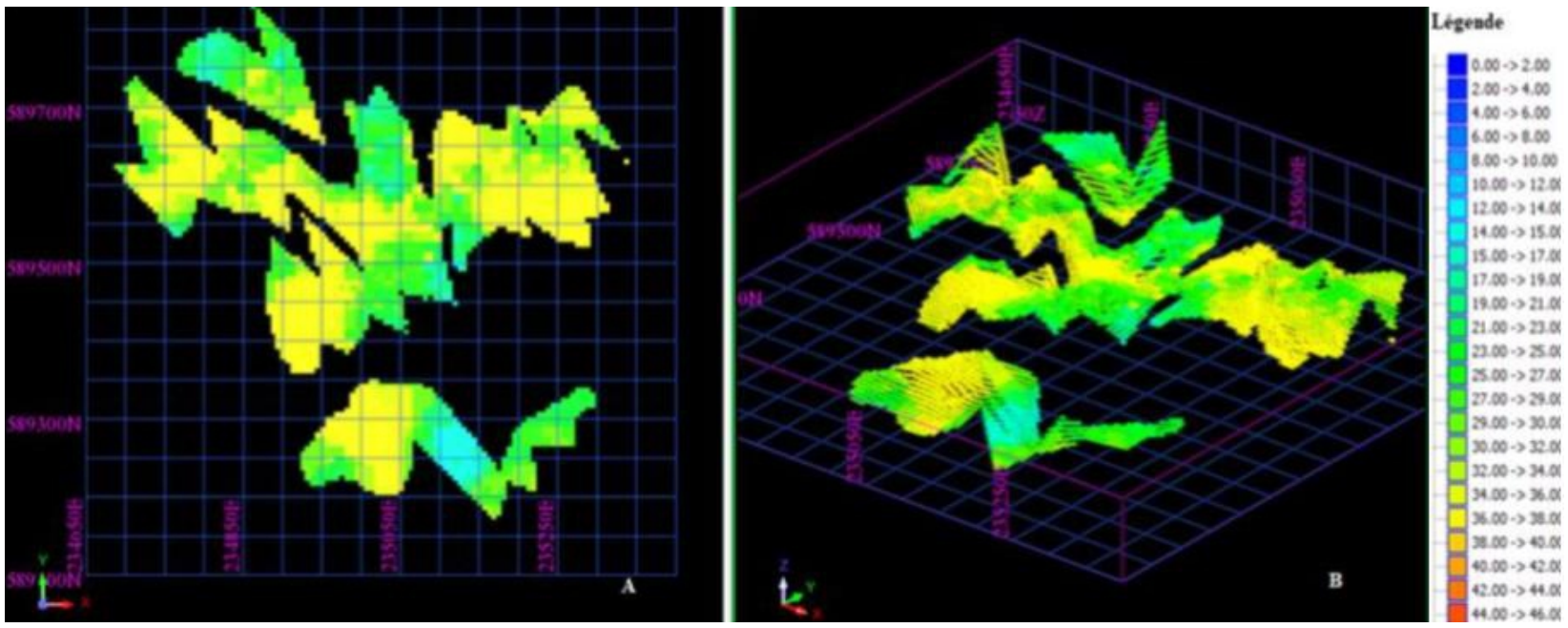

Figure 11 : Bloc modèle contraint estimé par Krigeage ordinaire

A) vue dans le plan $X Y, B$ ) vue en perspective

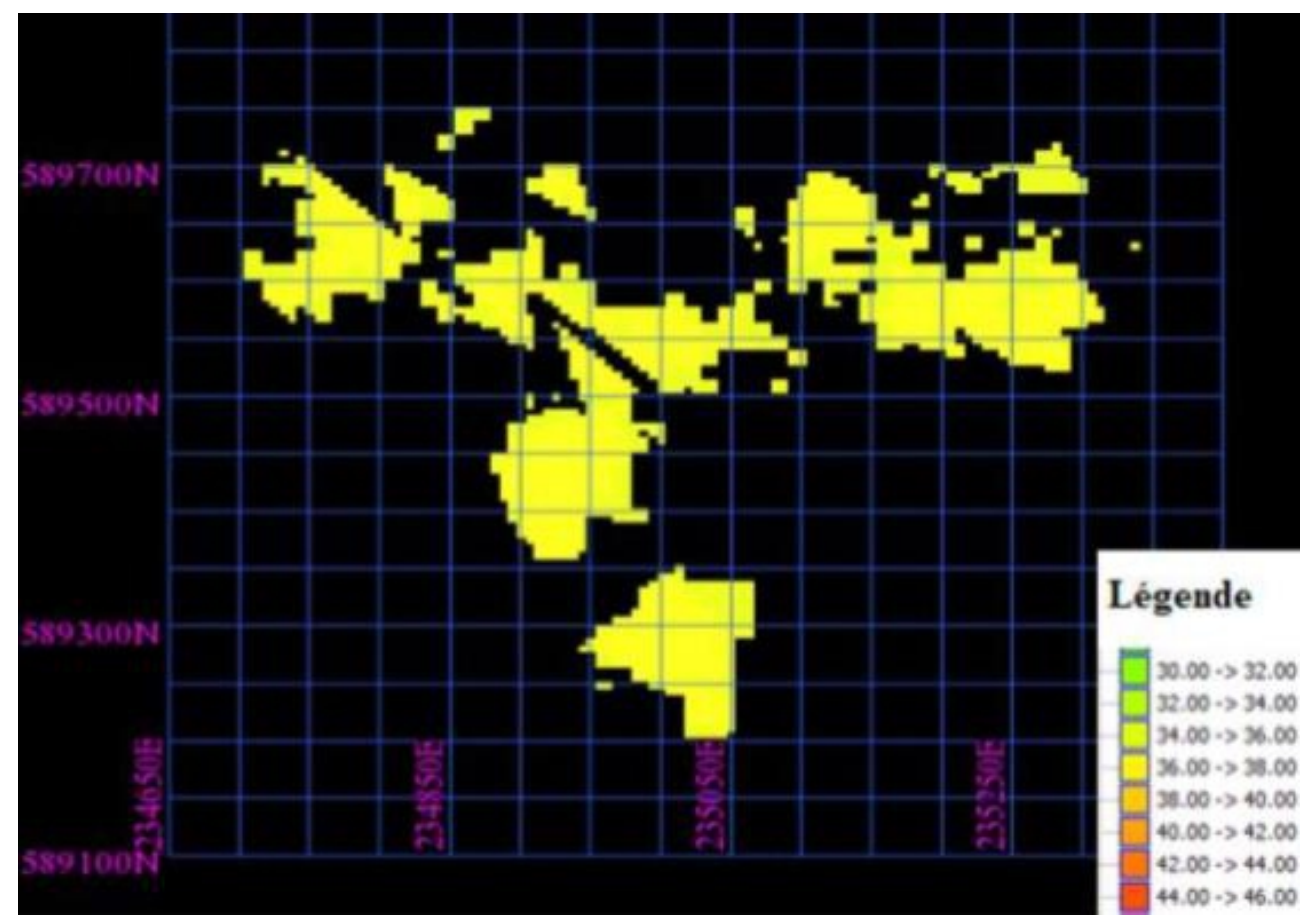

Figure 12 : Bloc modèle contraint estimé par krigeage ordinaire $(\mathrm{Tc} \geq 30 \%)$ 
Tableau V : Ressources estimées par Krigeage ordinaire

\begin{tabular}{|c|c|c|c|}
\hline Teneur Mn $(\%)$ & Volume $(\mathrm{m} 3)$ & Tonnes $(\mathrm{t})$ & Teneur moyenne Mn $(\%)$ \\
\hline $15.0>20.0$ & 6582 & 11245 & 18,46 \\
\hline $20.0>25.0$ & 46953 & 80999 & 22,88 \\
\hline $25.0>30.0$ & 111846 & 199542 & 27,52 \\
\hline $\mathbf{1 5} \leq$ Tc $\leq \mathbf{3 0}$ & $\mathbf{1 6 5 3 8 1}$ & $\mathbf{2 9 1 7 8 6}$ & $\mathbf{2 5 , 8 8}$ \\
\hline $30.0>35.0$ & 117305 & 209619 & 32,51 \\
\hline $35.0>40.0$ & 32646 & 57799 & 36,32 \\
\hline $40.0>45.0$ & 117 & 207 & 40,48 \\
\hline Tc $\geq \mathbf{3 0 . 0}$ & $\mathbf{1 5 0 0 6 8}$ & $\mathbf{2 6 7 6 2 5}$ & $\mathbf{3 3 , 3 4}$ \\
\hline Grand Total & $\mathbf{3 1 5 4 4 9}$ & $\mathbf{5 5 9 4 1 1}$ & $\mathbf{2 9 , 4 9}$ \\
\hline
\end{tabular}

\subsubsection{Estimation des ressources par la pondération inverse de la distance}

La pondération inverse de la distance a permis d'obtenir les modèles interpolés de bloc contraints des teneurs de la zone B-Centre (Figure 13). Le modèle géologique présente essentiellement deux zones de teneur :

- une variante du vert (19\% à 32\%)

- une variante du jaune (32\% à $40 \%$ )

Les ressources ont été évaluées suivant les teneurs de coupure (Tc) : $15 \% \leq \mathrm{Tc} \geq 30 \%$ pour l'Entreprise Minière de Basse Teneur (EMBT) soit 242915 t pour une teneur moyenne de $25,43 \%$, et $\mathrm{Tc} \geq 30 \%$ pour la CML soit $301199 t$ pour une teneur moyenne de 34,34\%, (Tableau VI). Au total, les ressources sont estimées à $544115 t$ pour une teneur moyenne de $30,36 \%$. Les modèles de bloc contraints de teneur de coupure, Tc $\geq 30 \%$ qui intéressent la CML ont été réalisés (Figure 14). 

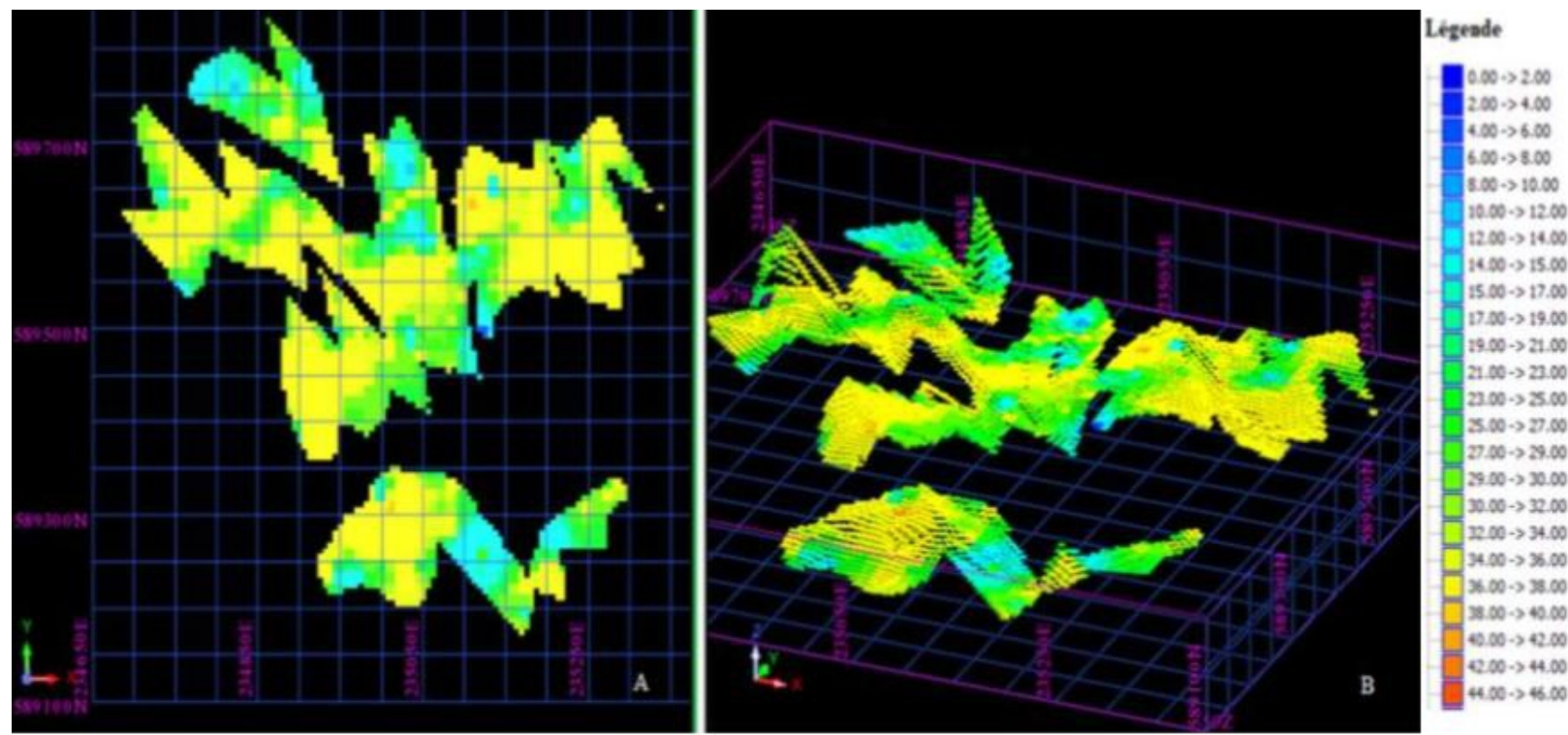

Figure 13 : Modèle de bloc contraint estimé par la pondération inverse de la distance A) vue dans le plan $\mathrm{XY}, \mathrm{B})$ vue en perspective

\subsubsection{Estimation des ressources par méthode du plus proche voisin}

La méthode du plus proche voisin a permis d'obtenir les modèles interpolés de blocs contraints des teneurs de la zone B-Centre (Figure 15). Le modèle géologique interpolé est très hétérogène avec une dominance de la variante du jaune (32 à 40\%). Les ressources ont été évaluées suivant les teneurs de coupure comprise entre $15 \%$ et $30 \%$ pour l'EMBT à $104547 \mathrm{t}$ pour une teneur moyenne de 23,97\%. Quant au teneur de coupure supérieur ou égale à $30 \%$ pour la CML, est estimée à $382864 t$ pour une teneur moyenne de $37,04 \%$, (Tableau VII). Au total les ressources sont estimées à 487411t pour une teneur moyenne de $34,23 \%$. Les modèles de bloc contraints de teneur de coupure, Tc $\geq 30 \%$ qui intéressent la CML ont été réalisés (Figure 16). 

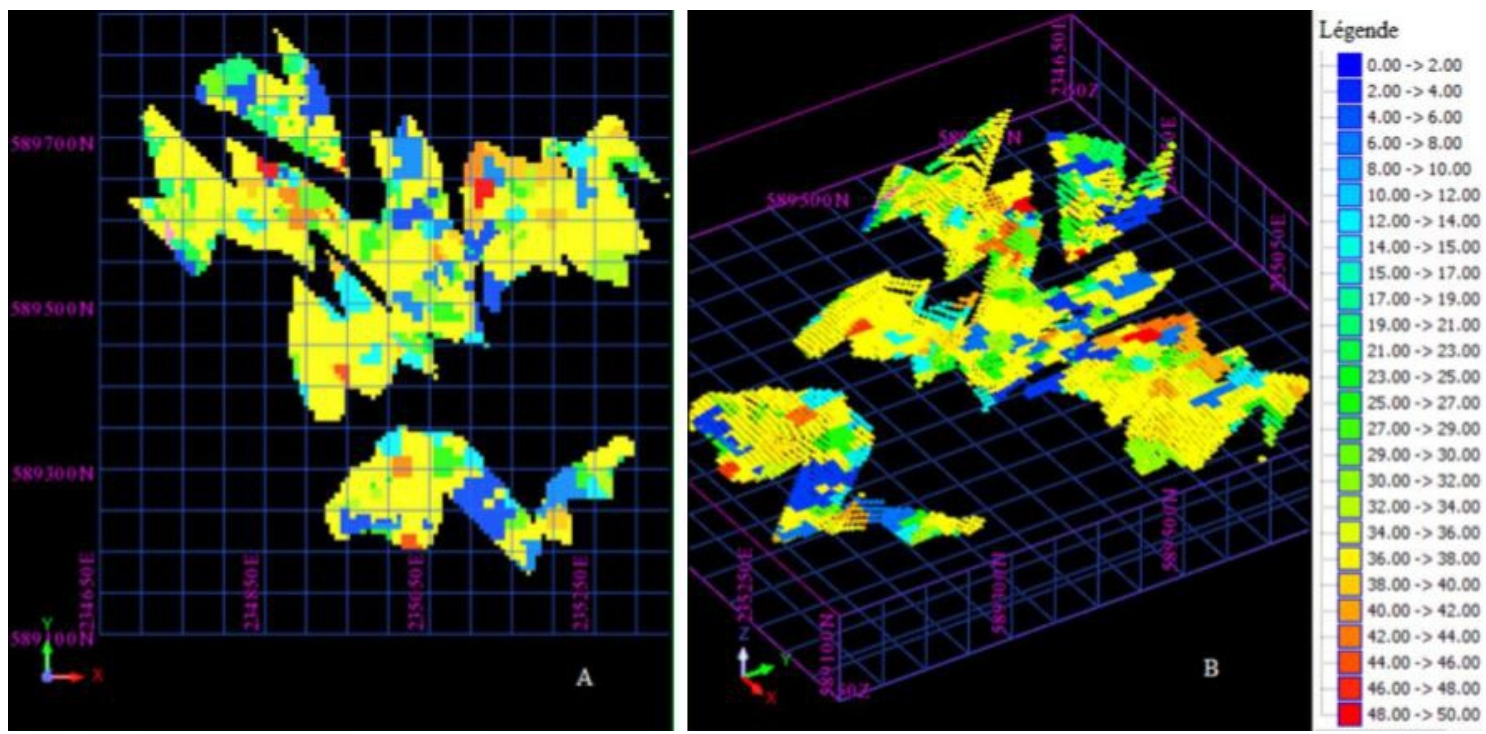

Figure 15 : Modèle de bloc contraint estimé par la méthode du plus proche voisin A) vue dans le plan $\mathrm{XY}$ B) vue en perspective

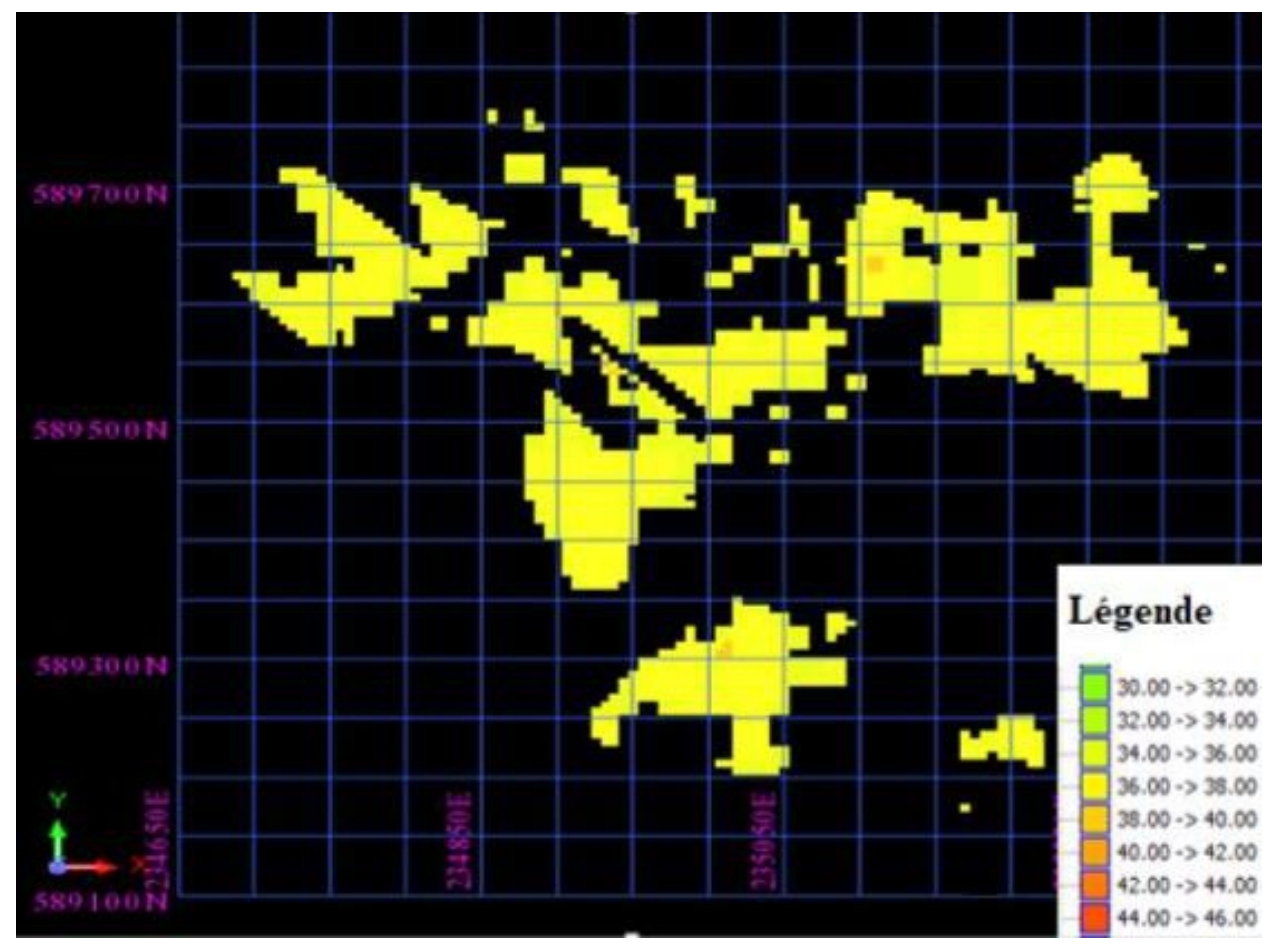

Figure 14 : Modèle de bloc contraint estimé par la pondération inverse de la distance pour $\mathrm{Tc} \geq 30 \%$ 
Tableau VII : Ressources estimées par la méthode du plus proche voisin

\begin{tabular}{|c|c|c|c|}
\hline Teneur Mn $(\%)$ & Volume $(\mathrm{m} 3)$ & Tonnes $(\mathrm{t})$ & Teneur moyenne Mn $(\%)$ \\
\hline $15.0>20.0$ & 15645 & 26493 & 17,77 \\
\hline $20.0>25.0$ & 35234 & 61139 & 22,85 \\
\hline $25.0>30.0$ & 86396 & 155283 & 27,76 \\
\hline $\mathbf{1 5} \leq$ Tc $\leq \mathbf{3 0}$ & $\mathbf{1 3 7 2 7 5}$ & $\mathbf{2 4 2 9 1 5}$ & $\mathbf{2 5 , 4 3}$ \\
\hline $30.0>35.0$ & 94131 & 167140 & 32,3 \\
\hline $35.0>40.0$ & 72627 & 128240 & 36,68 \\
\hline $40.0>45.0$ & 3145 & 5819 & 41,23 \\
\hline Te $\geq \mathbf{3 0 . 0}$ & $\mathbf{1 6 9 9 0 3}$ & $\mathbf{3 0 1 1 9 9}$ & $\mathbf{3 4 , 3 4}$ \\
\hline Grand Total & $\mathbf{3 0 7 1 7 8}$ & $\mathbf{5 4 4 1 1 5}$ & $\mathbf{3 0 , 3 6}$ \\
\hline
\end{tabular}

Les résultats des estimations donnent les différences des valeurs de tonnage (Tableau VIII) interpolés suivantes :

- La différence entre le Krigeage ordinaire et la pondération inverse de la distance est de l'ordre de $2,7 \%$;

- La différence entre le Krigeage ordinaire et la méthode du plus proche voisin est de l'ordre de $12 \%$;

- La différence entre la méthode du plus proche voisin et la pondération inverse de la distance est de l'ordre de $10 \%$.

Les différences minimes sont observées entre les méthodes de krigeage ordinaire et la pondération inverse de la distance.

Tableau VIII : Tableau récapitulatif des estimations par méthode d'interpolation

\begin{tabular}{|c|c|c|c|c|c|c|c|c|}
\hline \multirow{2}{*}{$\begin{array}{c}\text { Méthodes } \\
\text { d'interpolation }\end{array}$} & $\begin{array}{c}\text { Cubage } \\
(\mathrm{m} 3)\end{array}$ & $\begin{array}{c}\text { Tonnage } \\
(\mathrm{t})\end{array}$ & $\begin{array}{c}\text { Teneur } \\
\text { moyenne } \\
(\%)\end{array}$ & $\begin{array}{c}\text { Cubage } \\
(\mathrm{m} 3)\end{array}$ & $\begin{array}{c}\text { Tonnage } \\
(\mathrm{t})\end{array}$ & $\begin{array}{c}\text { Teneur } \\
\text { Moyenneb } \\
(\%)\end{array}$ & $\begin{array}{c}\text { Total } \\
\text { tonnage } \\
(\mathrm{t})\end{array}$ & $\begin{array}{c}\text { Teneur } \\
\text { moyenne du } \\
\text { gisement }(\%)\end{array}$ \\
\hline $\begin{array}{c}\text { Krigeage } \\
\text { ordinaire }\end{array}$ & 165381 & 291786 & 25,88 & 150068 & 267625 & 33,34 & 559411 & 29,44 \\
\hline $\begin{array}{c}\text { Pondération } \\
\text { inverse de la } \\
\text { distance }\end{array}$ & 137275 & 242915 & 25,43 & 150068 & 301199 & 34,34 & 544115 & 30,36 \\
\hline $\begin{array}{c}\text { Plus proche } \\
\text { voisin }\end{array}$ & 58467 & 104547 & 23,97 & 213759 & 382864 & 37,04 & 487411 & 34,23 \\
\hline
\end{tabular}

\subsubsection{Validations des méthodes d'estimations}

Les méthodes utilisées pour la modélisation et l'estimation des ressources dans cette étude ont été évaluées pour déterminer la meilleure méthode d'estimation. Le test statistique effectué en les comparant deux à deux pour les évaluer a fait ressortir que les méthodes de krigeage ordinaire et de la pondération inverse de la distance sont les plus performantes (tableau IX).

Les résultats des tests statistiques de comparaison montrent une forte corrélation $(0,7544)$ et un faible rapport de régression linéaire (IDW $=1,0394$ 
OK +/0,8070) entre les valeurs interpolées par la méthode du krigeage ordinaire et la pondération inverse (Figure 17) tandis que ces dernières comparées à la méthode du plus proche voisin donnent une faible corrélation et un rapport de régression linéaire élevé (Figure 18 et 19).

Tableau IX : Comparaison des méthodes d'estimation deux à deux

\begin{tabular}{|c|c|c|}
\hline Méthodes comparées & Régression linéaire & $\begin{array}{c}\text { Coefficient de } \\
\text { corrélation }\end{array}$ \\
\hline $\begin{array}{c}\text { Krigeage ordinaire (OK) vs Plus proche } \\
\text { voisin (PV) }\end{array}$ & $\mathrm{PV}=1,1980$ OK +/- 4,8796 & 0,4527 \\
\hline $\begin{array}{c}\text { Krigeage ordinaire (OK) vs } \\
\text { Pondération inverse de la distance } \\
\text { (IDW) }\end{array}$ & IDW $=\mathbf{1 , 0 3 9 4}$ OK +/0,8070 & $\mathbf{0 , 7 5 4 4}$ \\
\hline $\begin{array}{c}\text { Plus proche voisin (PV) vs Pondération } \\
\text { inverse de la distance (IDW) }\end{array}$ & IDW $=0,3829$ PV + 18,0140 & 0,7355 \\
\hline
\end{tabular}
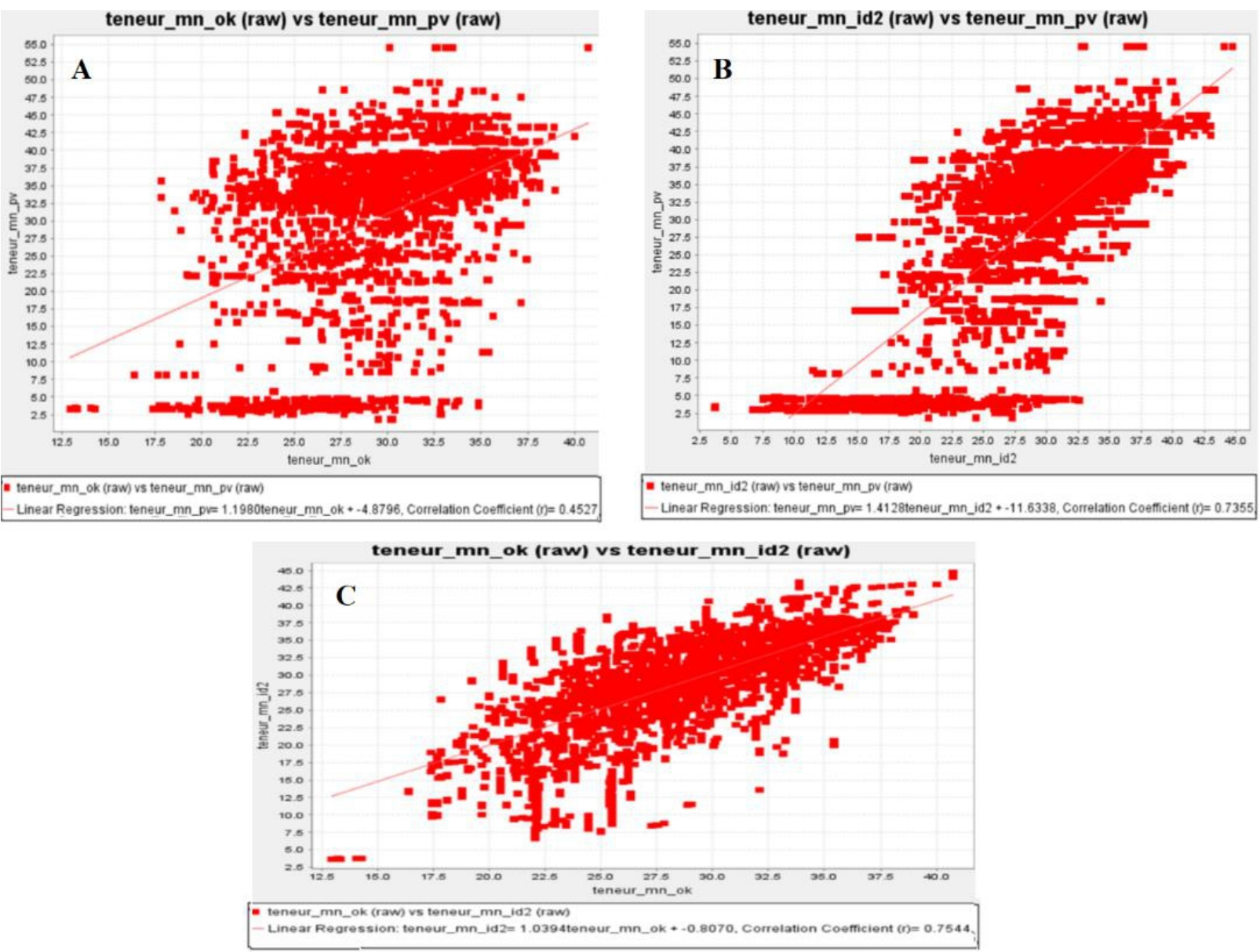

Figure 17 : Test statistique : A) Test statistique du Krigeage ordinaire vs Plus proche voisin,

B) Test statistique de la Pondération inverse de la distance vs Plus proche voisin,

C) krigeage ordinaire vs pondération inverse de la distance 


\subsection{Discussion}

Le modèle solide obtenu donne trois corps minéralisés indépendants de forme lenticulaire et orientés suivant la direction birrimienne NE-SW avec de faibles pendages (10 à 15\%). Ces lentilles sont observées près de la surface topographique, ce qui s'accorde avec l'hypothèse de Grandin (1976) selon laquelle l'ensemble de la série se présente sous un faciès lenticulaire, les zones minéralisées sont reparties dans la partie haute du versant d'une chaine de collines allongée selon la direction birrimienne. Selon cet auteur, ces lentilles doivent donc être considérées comme indépendantes. Ceci s'expliquerait selon l'hypothèse d'Arnould (1957) par un dépôt de sédiments manganésifères en milieu calme éventuellement peu profond du type lacs ou lagunes formant un chapelet de petits bassins. Les inclinaisons faibles sont rencontrées selon Grandin (1976) près des ruptures de pentes et de façon générale à proximité de la surface du sol (partie superficielle de B-centre). Les tests statistiques ont montrés que la méthode géostatistique probabiliste du krigeage ordinaire est la plus performante des trois méthodes et donc la plus adaptée pour l'estimation des ressources de la zone B-centre. Elle donne des résultats plus proches de la réalité que les méthodes déterministes de la pondération inverse de la distance et du plus proche voisin. Toutefois, des deux méthodes déterministes, la méthode de la pondération inverse de la distance ne présente pas de différences notables avec le krigeage ordinaire (Weber et Englund, 1994). Le coefficient de corrélation élevé entre les deux méthodes déterministes s'expliquerait par le fait que ces méthodes ont en commun de prendre en compte uniquement la distance qui sépare les sites entre eux. Cela donne un poids plus important aux groupements de données, alors que cela n'est pas nécessairement justifié (Despagne, 2006). Par ailleurs, à la différence de la méthode du plus proche voisin, l'influence relative d'un point d'observation dans la pondération inverse de la distance diminue avec la distance, le séparant de l'endroit où il faut effectuer une estimation de la même variable. Ce qui explique donc le rapport élevé de régression linéaire entre ces deux méthodes. Les erreurs commises par la méthode du plus proche voisin sont alors plus élevées que celles produites par la pondération inverse de la distance (Ariane \& Diane, 2010). La pondération inverse de la distance fonctionne selon le principe géographique d'après lequel les objets rassemblés tendent à se ressembler d'avantage. La méthode d'interpolation du krigeage ordinaire par contre qui est une méthode géostatistique, utilise l'arrangement spatial de l'ensemble des points échantillonnés en plus d'accorder un poids à la distance entre les points (McCoy \& Johnston, 2001). Cette particularité fait en sorte que le krigeage ordinaire produit des erreurs plus faibles probablement en raison de l'échantillonnage le long des transects. En effet, cette méthode est à mesure de déterminer, contrairement aux deux autres méthodes la tendance entre les transects, c'est-à-dire la relation spatiale entre les données. 
Selon Ariane et Diane (2010), McCoy et Johnston (2001), le krigeage ordinaire serait la méthode d'estimation linéaire garantissant le minimum de variance. La différence fondamentale des méthodes d'interpolation déterministes d'avec le krigeage ordinaire est que ce dernier tient compte à la fois de l'information relative à leur position et du caractère aléatoire du phénomène étudié. De plus, elle permet d'intégrer des informations auxiliaires dans l'estimation. Ces avantages font considérablement améliorer les estimations dans le contexte spatial. La mise en œuvre du krigeage passe par une analyse des données (variogramme). Le modèle 2D ne permet pas le stockage implicite des informations qui représentent la troisième dimension (hauteur). La modélisation d'environnements géologiques en trois dimensions était donc un objectif à atteindre pour la CML. Ces modèles 3D sont des représentations de la réalité dans laquelle les objets modélisés ont une position $(\mathrm{X}, \mathrm{Y}, \mathrm{Z})$ et qui peuvent contenir des objets ponctuels, linéaires, surfaciques et volumiques (McGaughey \& Morrison, 2001).

\section{Conclusion}

De la modélisation et de l'estimation des ressources de la zone Bcentre on peut retenir que le gisement est composé de trois corps minéralisés lenticulaires indépendant de faible pendage (10 à 15\%) d'orientation générale birrimienne (NE-SW), peu profond, ne dépassant pas $8 \mathrm{~m}$ d'épaisseur. L'emploi des méthodes déterministes et géostatistique probabiliste pour l'estimation des ressources et les tests statistiques de comparaison de méthodes ont permis de retenir que le krigeage ordinaire est la plus appropriée pour l'estimation et présente l'avantage d'étudier la distribution spatiale (variogramme) de la minéralisation. Les ressources sont donc estimées pour les teneurs de coupure de l'EMBT $(15 \% \leq \mathrm{Tc} \leq 30 \%)$ à $291787 \mathrm{~T}$ pour une teneur moyenne de $25,87 \%$ et pour la CML (Tc $\geq 30 \%$ ) à $267624 \mathrm{~T}$ pour une teneur moyenne de $33,33 \%$. Les ressources totales ont donc été estimées à 559411 pour une teneur moyenne de 29,44\%. Par ailleurs, la modélisation 3D utilisée dans cette étude présente bien plus d'avantage que la 2D utilisée par la CML. D'abord la 3D permet une visualisation dans l'espace tridimensionnel et la représentation d'objet volumique. De plus, elle contribue à l'analyse des relations spatiales entre les objets et à l'analyse géostatistique. Enfin, la modélisation 3D permet l'estimation directe des ressources, et la réduction des coûts d'exploration et d'exploitation du gisement tout en diminuant les risques financiers et matériels. Elle facilite les échanges d'information et la communication entre les différents spécialistes. Au regard de tous les travaux exécutés, les résultats de modélisation et d'estimation obtenus donnent un bon espoir quant à l'utilisation de la 3D et des méthodes géostatistiques dans le processus de certification des ressources de la CML. En perspective il parait important pour la confirmation et l'amélioration des 
résultats de la présente étude, de mener une étude de faisabilité pour évaluer la rentabilité du gisement et aussi de suivre les travaux d'exploitation future de la zone B-centre.

\section{References:}

1. Adrian D. \& Diane S.L. (2010). Comparaison des méthodes d'interpolation pour l'élaboration de modèle numérique d'élévation de haute précision dans la représentation microtopographique des plaines inondables. Hydrological sciences jounal - Journal des sciences hydrologiques, 55(4) : 526-539.

2. Arnould M. (1957). Note sur le gisement de manganèse de GrandLahou (Côte d'Ivoire). Rapport d'étude, 5 p.

3. BRGM (1984). Ressources minières française, Les gisements de manganèse (situation en 1981), Rapport d'étude du BRGM, France, $137 \mathrm{p}$.

4. Brodaric B., Gahegan M. \& Harrap M.R. (2004). The art and science of mapping: computing geological categories from field data. Computers \& Geosciences, 30 : 719-740.

5. Dago A.G.B. (2014). Pétrogénèse du gisement Manganésifère de Lauzoua dans le département de Guitry (Sud de la Cote d'Ivoire). Mémoire de Master en Pétrologie- Métallogénie, UFR-STRM, Université Félix Houphouët Boigny de Cocody, Côte d'Ivoire, 94p.

6. Delor C., Diaby I., Simeon Y., Yao B., Akre D., Okou A., Konate S., Tastet J-P., Vidal M., Traore I., Dommanget A., Cautru J-P., Konan G. \& Chiron J-C. (1995). Carte géologique de la Côte d'Ivoire au 1/200 000 ; Feuille de Grand-Lahou.

7. Despagne W. (2006). Méthode géostatistique pour l'interpolation et la modélisation en 2D/3D des données spatiales. Mémoire de Master en statistique et informatique, Université de Bretagne Sud (France), 145 p.

8. Grandin G. (1976). Aplanissements cuirassés et enrichissement des gisements de manganèse dans quelques régions d'Afrique de l'ouest. Mémoire de master option sciences de la terre, Université de Strasbourg, France 60 p.

9. McCoy J. \& Johnston K. (2001). Using ArcGis Spatial Analyst. Redlands, CA: ESRI Press, 194 : 41-46.

10. McGaughey J. \& Morrison K. (2001). The common earth model : A revolution in exploration data integration. Mira Geoscience, 11 (2) : 310.

11. Papon A. (1962). Problème du manganèse. Régions de Grand-Lahou, Divo, Toumodi. BRGM, Abidjan, multigr. 26 p. 
12. Weber D.D. \& Englund E.J. (1994). Evaluation and comparison of spatial interpolators II 4 International Association for Mathematical Geology vol. 24. no. 4. 